\title{
Improving medication adherence in stroke survivors: mediators and moderators of treatment effects
}

${ }^{1}$ Ronan E. O’Carroll, ${ }^{1}$ Julie A. Chambers, ${ }^{2}$ Martin Dennis, ${ }^{2}$ Cathie Sudlow, ${ }^{3}$ Marie Johnston

${ }^{1}$ Psychology, School of Natural Sciences, University of Stirling, Stirling, Scotland, UK;

${ }^{2}$ Division of Clinical Neurosciences, Western General Hospital, Edinburgh and University of

Edinburgh, Edinburgh, Scotland, UK; ${ }^{3}$ Aberdeen Health Psychology Group, University of Aberdeen, Aberdeen, Scotland, UK

Correspondence to: Prof. Ronan O’Carroll, Psychology, School of Natural Sciences, University of Stirling, Stirling, FK9 4LA. Email: reo1@stir.ac.uk

Key words: Stroke, adherence, implementation intentions, medication beliefs, antihypertensives

This article may not exactly replicate the final version published in the APA journal. It is not the copy of record. 


\begin{abstract}
Objective: The purpose of the current study was to test theory-based predictions of mediators and moderators of treatment effects of a pilot randomised controlled trial which aimed to increase adherence to preventive medication in stroke survivors via addressing both automatic (i.e. habitual responses) and reflective (i.e. beliefs and value systems) aspects of medication-taking behaviour.
\end{abstract}

Methods: Sixty-two stroke survivors were randomly allocated to either an Intervention or Control group. Intervention participants received a brief two-session intervention aimed at increasing adherence via a) helping patients establish better medication-taking routines using implementation intentions plans (automatic) and b) eliciting and modifying any mistaken patient beliefs regarding medication and/or stroke (reflective). The Control group received similar levels of non-medication-related contact. Primary outcome was adherence to antihypertensive medicine measured objectively over 3 months using an electronic pill bottle. Secondary outcome measures included self-reported adherence (including forgetting) and beliefs about medication.

Results: Intervention participants had 10\% greater adherence on doses taken on schedule (intervention $97 \%$; control $87 \%, 95 \%$ CI $(0.2,16.2), p=.048)$, as well as significantly greater increases in self-reported adherence and reductions in concerns about medication. Treatment effects were mediated by reductions in both forgetting and concerns about medication; and moderated by the presence of pre-existing medication-taking routines.

Conclusions: Addressing both automatic and reflective aspects of behaviour via helping stroke survivors develop planned regular routines for medication-taking and addressing any concerns or misconceptions about their medication can improve adherence and thus potentially patient outcomes. 


\section{Introduction}

Stroke is the fourth highest cause of mortality in the United States, and has been estimated to account for around 1 in every 18 deaths, with total U.S. direct and indirect economic costs of over $\$ 34$ billion per annum in 2008 ; it is also a leading cause of disability, including mobility and speech problems, which frequently result in dependency in everyday activities (Roger et al., 2012). People who have a stroke or transient ischaemic attack (TIA) have a $30-43 \%$ risk of recurrent stroke within 5 years (Warlow et al., 2008), and hence an increased risk of further disability and reduced quality of life. This risk can be substantially reduced by taking combined preventive medication over the long-term (e.g. a combination of cholesterol lowering drugs, blood thinning drugs and anti-hypertensive medication can provide an absolute risk reduction of $8.5 \%$ in the first year and $4.1 \%$ annually for subsequent years) (Sudlow, 2008).

Adherence to medication in a timely manner is a primary determinant of treatment success. Despite this, it is estimated that in developed countries, only $50 \%$ of patients who suffer from chronic diseases adhere to treatment recommendations (WHO, 2003). In particular, adherence to preventive medication in stroke survivors is often poor (Bushnell, Zimmer, Pan, Olson, \& Zhao, 2010), despite its evident success at reducing the risk of future strokes. Non-adherence to medication may be either non-intentional or intentional (Clifford, Barber, \& Horne, 2008). Non-intentional non-adherence represents a patient's failure to actively remember, or in some cases physically manage, to take their medicine as prescribed. This frequently depends on their ability to develop adequate habitual routines, and is associated with automatic behaviour, triggered by environmental cues. Intentional nonadherence is associated with beliefs about illness and treatment, particularly the perceived effectiveness and necessity of medication versus concerns about possible harmful effects, and represents a reflective choice of how to behave (Strack \& Deutsch, 2004). 
Temporal self-regulation theory (Hall \& Fong, 2007) can help explain why many patients with chronic conditions do not achieve optimal medication adherence, as it can account for both non-intentional and intentional non-adherence. This theory attempts to incorporate both automatic (based on past experience or environmental cues) and reflective (based on beliefs or value systems) behavioural processes, alongside consideration of the short-term cost versus long-term gain, in order to explain why individuals do not always behave in a manner which will support their long-term health goals. Taking medication can be associated with immediate costs (e.g. having to obtain medication, side-effects) which are offset against longer-term gains. Patients' beliefs in the efficacy of their medication may impact on their assessment of longer term benefits, especially as many chronic conditions, such as high blood pressure, are not associated with immediate symptoms; for example, patients with hypertension may choose not to take medication as they do not feel ill, and therefore conclude they are 'healthy' (Anthony, Valinsky, Inbar, Gabriel, \& Varda, 2012), or because they see the condition as temporary (Meyer, Leventhal, \& Gutmann, 1985). Patients' adherence may also be affected by their motivation to reduce the short-term costs of taking medication. For example, patients often seek to minimise their intake of medicines, in part to reduce the risk of negative consequences such as side-effects (Pound et al., 2005). Temporal self-regulation theory posits that whilst behaviour is primarily a function of the perceived likelihood of expected outcomes and the values attached to them, it is moderated by both behavioural pre-potency (reflecting past performance (i.e. automatic or habitual responses) or environmental cues to action) and self-regulatory capacity (i.e. an individual's ability to regulate their own behaviour). Both of these may affect patients' ability to develop and adhere to adequate medication-taking routines which will in turn impact on the regularity of their medication-taking.

Both non-intentional and intentional aspects are likely to play a part in non-adherence to 
medication in stroke survivors. Non-intentional non-adherence (e.g. forgetting) is likely in this group, as age is a major risk factor for stroke, and stroke often causes cognitive impairment (such as memory loss), and even mild cognitive impairment has been associated with poor medication adherence in elderly populations (Hayes, Larimer, Adami, \& Kaye, 2009). As well as its effect on a patient's ability to develop habitual medication-taking routines, cognitive impairment may also impact on patient's judgement about the benefits of taking their medication. Elderly stroke survivors are likely to have complex medication regimes, and so any negative beliefs they hold about treatment and its consequences may lead to higher intentional non-adherence in this group. A recent study on determinants of adherence in stroke survivors supported temporal self-regulation theory: it identified that patients' immediate concerns about their medication (e.g. that they would become dependent, or suffer serious side effects), and a lesser belief in its potential benefits were both related to poorer adherence (O’Carroll et al., 2011). Further, those patients who had developed automatic medication-taking routines were likely to have higher adherence overall (Chambers et al., 2011).

Preventing stroke reoccurrence by increasing adherence to secondary preventative medication may improve quality of life for individuals and will help cut healthcare costs and so is an important focus for intervention. Whilst some behavioural change techniques address both reflective and automatic components of behaviour, many do not. A review of existing interventions aimed at increasing adherence concluded that current studies have had only limited success and that more research is needed on combined approaches involving multiple strategies, as these have been most effective (Haynes, Ackloo, Sahota, McDonald, \& Yao, 2008).

The current intervention was designed to address both reflective and habitual aspects of adherence behaviour in a brief, personalised intervention in stroke survivors. Reflective 
behaviour (represented by intentional non-adherence) was targeted by examining and modifying any mistaken beliefs that patients may have about their medication and/or stroke, using a model which has proved successful in improving patients' views of their illness, and led to better rehabilitation after myocardial infarction (Petrie, Cameron, Ellis, Buick, \& Weinman, 2002). Habitual behaviour (represented by non-intentional non-adherence) was addressed by the use of a simple If-then plan (Implementation Intentions) (Gollwitzer, 1999), to be used by each patient as an aid to developing automatic routines for medication-taking, thereby reducing cognitive load and hence forgetting. Implementation intentions formation may be viewed as a self-regulatory tool which has been shown to be effective at increasing adherence to health-behaviours in older adults over sustained periods of time and may be particularly helpful in stroke survivors as it does not seem to be affected by age-related cognitive decline (e.g. Liu \& Park, 2004). However, forming deliberate intentions to perform a behaviour may be obstructed by the existence of previous habits or cues (Lally \& Gardner, 2011). Habit strength has been shown to moderate the effect of Implementation Intentions on change of negative health behaviours, for example where Implementation Intentions were found to reduce smoking only for those with weak initial smoking habits (Webb \& Sheeran, 2009). Thus with regard to medication adherence, it may be harder to persuade patients with ingrained habits for medication-taking, even if ineffective, to form or adopt an Implementations Intentions plan, which aims to change their established routines. In addition patients already using effective environmental cues may not gain any additional benefit from the development of a formalised Implementations Intentions plan.

Both Implementation Intentions and belief modification have been shown to be helpful in increasing adherence to medication in other health conditions (e.g. Alhalaqia, Nawafleh, Clark, \& Gray, 2012; Brown, Sheeran, \& Reuber, 2009), but, to our knowledge, have never been used in combination in stroke survivors. The aims of this study, as stated in the study 
protocol article (O’Carroll, Dennis, Johnston, \& Sudlow, 2010), were to test whether this brief intervention would improve both intentional and non-intentional adherence to medication and to examine whether increased adherence would be mediated by changes in the behaviours targeted for change (i.e. reduced forgetting and reduced medication concerns). An additional aim was to test for any moderating effect of previous habits (i.e. existing environmental cues) on adherence; the hypothesis is that the intervention would be more effective for those with weak pre-existing habits (i.e. environmental cues).

\section{Methods}

Full details of methods for this pilot randomised controlled trial are reported in the protocol article (O'Carroll et al., 2010) and the simple treatment effects article (O'Carroll, Chambers, Dennis, Sudlow, \& Johnston, 2013), in compliance with CONSORT (CONsolidated Standards of Reporting Trials) guidelines (Moher et al., 2010).. In this article only methods which are pertinent to the mediator and moderator analyses are reported.

\section{Participants}

Participants were recruited from consecutive discharges at the stroke unit at the Western General Hospital in Edinburgh. Inclusion criteria were first stroke or TIA, discharged to home, and taking any preventive stroke medication. All participants were white Caucasian and all gave written informed consent to take part. Ethical approval was granted by Lothian NHS Board, South East Scotland Research Ethics Committee.

\section{Measures}

\section{MEMS (Medication Event Monitoring System, MEMS® Aardex Ltd, Switzerland)}

The primary outcome measure was electronically recorded openings using MEMS pill bottles, used in both treatment arms for 3 months. Following Brown et al. (2009), three variables were calculated i.e. a) percentage of doses taken (versus prescribed), b) percentage of days on which the correct dose was taken and c) percentage of doses taking on schedule 
i.e. within $+/-3$ hours of the median time taken.

The Medication Adherence Report Scale (MARS) (Horne, 2004)

The self-report MARS consists of 5 items, each representing a different aspect of medication taking. We calculated a total score (all 5 items, possible range 5-25) and separate scores for non-intentional non-adherence (i.e. item 1: forgetting, possible range 1-5) and intentional non-adherence (i.e. items 2-5, possible range 4-20). Higher scores indicate higher adherence. The MARS has been used extensively to measure adherence in patients with chronic diseases and has shown good reliability (internal and test-retest) and validity (convergent and criterion) (Horne, 2004). Internal consistency (Cronbach's $\alpha$ ) for this study was .71 for the total score and .79 for the intentional non-adherence subscale.

The Beliefs about Medication Questionnaire (BMQ) (Horne \& Weinman, 1999)

The two, 5-item BMQ-Specific subscales were used in the current study. These represent: a) beliefs about the necessity of a patient's medication (Necessity) and b) concerns/beliefs about the risks and/or negative effects of taking their medication (Concerns). Higher scores equate to higher necessity and higher concerns. The BMQ-specific subscales have shown good reliability and validity amongst patients with varied illnesses including heart conditions, asthma and diabetes (Horne, Weinman, \& Hankins, 1999). Internal consistency (Cronbach's $)$ was .84 for BMQ-necessity and .76 for BMQ-concerns.

The Brief Illness Perception Questionnaire (BIPQ) (Broadbent, Petrie, \& Weinman, 2004)

The BIPQ consists of 8 separate items (consequences, timeline, personal control, treatment control, identity, illness concern, coherence (understanding) and emotional representation) relating to the patient's illness (their stroke or TIA), each scored from 1-10, therefore internal reliability statistics are not appropriate. Higher scores indicate a greater belief in each construct (e.g. higher belief that a patient's treatment can control their illness (treatment 
control); a belief that their illness will last for a longer period (timeline)). The BIPQ provides an easy-to-complete, psychometrically robust measure of the major components of illness perceptions (Broadbent et al., 2004).

Perception of benefits (Trewby et al., 2002)

Following Trewby et al. (2002) and a previous study with stroke survivors (O'Carroll et al., 2011), participants were asked to indicate their perception of the benefits (0-100\%) provided by their stroke medication over the next five years (i.e. a single item).

Mini-Mental State Examination (MMSE) (Folstein, Folstein, \& McHugh, 1975)

The MMSE is a brief, valid and reliable instrument which has been extensively used to detect cognitive impairment in both research and clinical settings, including stroke research. Correct responses are totalled to give a maximum possible score of 30 . The aim was to exclude participants with cognitive difficulties which could affect study participation. To this end the conventional cut-off score of 23 was used, as no elderly psychiatrically healthy control subject scored $<24$ in the initial study (Folstein et al., 1975). All participants in the current study scored 26 or higher, thus there were no exclusions using this criterion.

\section{Procedure}

Patients were screened for the brief intervention by completing a questionnaire around 3 months after discharge. Inclusion criteria were: less than maximum MARS scores (i.e. $<25)$, and taking at least one anti-hypertensive medication. Exclusion criteria were: receiving help in taking medications or using a pharmacy-supplied Dosette box (where tablets are pre-sorted into daily compartments). A power calculation indicated a sample size of $n=30$ in each treatment arm would detect a medium effect on MEMS adherence.

Two brief sessions, two weeks apart were conducted by a trained Research Fellow for both the Intervention and Control groups, either in the participant's home or at the Wellcome 
Trust Clinical Research Facility at the Western General Hospital; travel expenses were reimbursed in the latter case.

\section{Intervention condition}

Session 1 (Implementation Intentions). The main focus of Session 1 was to address automatic aspects of medication-taking behaviour by helping each patient develop an effective medication-taking routine using an Implementation Intentions approach. This approach corresponds to items 1.4 'Action Planning' and 7.1 'Prompts/Cues' in the taxonomy for behaviour change techniques developed by Michie et al. (2013).

Before formulating the plan, participants were asked to describe how they currently took their anti-hypertensive medication. This was audio-taped, and following Session 1, the Research Fellow coded whether the participant had mentioned a time, place, or activity (each as yes or no) in their description of medication-taking (e.g. 'around 8 o'clock in the morning with my breakfast' would constitute a time and activity). The number of 'yeses' was then totalled to give 'number of freely mentioned II (Implementation Intentions) cues' (range 0-3), which was used in the moderator analysis.

Participants were then asked to formulate an Implementation Intentions plan by writing down exactly when and where they would take their medication, using the format of an ifthen plan i.e. 'If it is time $\mathrm{X}$ in place $\mathrm{Y}$ and $\mathrm{I}$ am doing $\mathrm{Z}$, then I will take my blood pressure pill' by linking their medication-taking to something that they did every day (e.g. 'If it is 8 am and I am in the bathroom and I am about to brush my teeth, then I will take my blood pressure tablet'). Participants were helped to complete an individualised worksheet plan for each scheduled daily dose of their primary anti-hypertensive medication, and then to read this through until they could remember it without looking at the written plan.

Session 2 (Modifying dysfunctional beliefs).

The Implementation Intentions plan was reviewed at the beginning of Session 2 and any 
required changes were made following the methods described by Sniehotta (Sniehotta, Scholz, \& Schwarzer, 2006). Session 2's main focus was to address reflective aspects of behaviour by eliciting and questioning any mistaken illness and/or medication beliefs. Responses on the BIPQ and BMQ were used as the basis for eliciting beliefs regarding a participant's medication (e.g. beliefs regarding side-effects, interactions between medicines etc.) and/or regarding their stroke (e.g. underlying risk factors, long-term effects).

Participants were not told their scores on the BMQ and BIPQ, rather these were used to guide questions regarding potential issues, particularly if the participant did not freely volunteer these at the interview (e.g. if the participant reported that they didn't mind taking medication, but their score on the BMQ question 'I sometimes worry about the long-term effects of my medicines...' was 'agree' or 'strongly agree', the researcher would ask 'Do you ever worry about the long-term effects of taking your medication'). The Research Fellow then aimed to provide evidence which would help participants come to believe that the necessity of their medication was more important that any concerns they had about their prescribed medication (Horne \& Weinman, 1999). For example, if a patient expressed concerns about becoming physically dependent on their anti-hypertensive medication; the Research Fellow would provide information and give reassurance about the lack of evidence for any dependency effects. This approach corresponds to items 5.1 'Information about health consequences' and 5.2 'Salience of consequences' in the taxonomy for behaviour change techniques reported by Michie et al. (2013).

\section{Control condition}

Participants in the Control group also received visits from the Research Fellow at Session 1 and 2. During these sessions, the Research Fellow talked in general, non-medication related terms to the participant about their stroke and its aftermath (e.g. how they and their family had coped) in order to control for any effects of attention or social contact. 


\section{Both groups}

The MEMS pill bottle was filled on a monthly basis with the patient's own anti-hypertensive medication and MEMS data was recorded from the day after Session 2 for 3 months. Followup was the same in both groups and data on all measures were collected at the same time points for both treatment arms. For each of the next two months (Sessions 3 and 4), the Research Fellow made a brief visit to refill the MEMS pill bottle and download an electronic reading from the MEMS cap onto a laptop PC. The BIPQ, BMQ and perceptions of medication benefits measures were also completed by the participant at the first of these visits. At 3-months (Session 5), the Research Fellow made a final visit to take a last MEMS cap reading and collect the MEMS pill bottle. The participant also completed a final version of the outcome measures.

\section{Randomisation}

Randomisation to either the Intervention or Control arm was carried out independently by the Edinburgh Clinical Trials Unit, using a minimisation algorithm. Participants were blinded to treatment allocation.

\section{Training of Research Fellow}

The Research Fellow is a UK registered Health Psychologist with over 10 years' experience of working in applied settings. She was trained in the intervention by the Principal Investigator, who had been previously trained in the intervention used by Petrie (Petrie, Cameron, Ellis, Buick, \& Weinman, 2002) in eliciting and addressing mistaken beliefs. Both the intervention and control group sessions (1 and 2) were piloted via role-play with the Principle Investigator acting as a pseudo-patient. These sessions were video-recorded, and feedback was given to the Research Fellow, after which minor modifications were made to the procedure. 


\section{Statistical Analysis}

An a priori decision had been made to exclude all participants with no data on the primary outcome measure (the MEMS pill bottle) from the analysis, in accordance with the published protocol (O’Carroll et al., 2010). Four people randomised to treatment were excluded from the analysis as they did not use the MEMS pill bottle (see Supplemental Figure S1: three became ineligible before starting the intervention and the other refused the use of the MEMS bottle at session 2); 58 patients were therefore analysed as allocated. A further 4 people were lost to follow-up (Supplemental Figure S1); these four are included in the analyses. Dropouts and/or those with missing data did not differ by treatment group $\left(\chi^{2}(2)=0.5, p=.766\right)$, nor on any of the pre-treatment outcome measures, gender, Scottish Index of Multiple Deprivation (SIMD), MMSE scores or overall MEMS scores (data available from the authors).

Missing data was addressed by using multiple imputation, via ICE (Imputation of Chained Equations) in the Stata software package. Five datasets were created for pooled analysis and test statistics were pooled using Rubin's rules (Rubin, 1987). All other analysis was carried out in SPSS version 19: t-tests and $\chi^{2}$ were used to test basic differences between treatment groups; Pearson's r was for first-order correlations, with the exception of MARS scores (which had ceiling effects), where Spearman's rho was used. Repeated measures Analysis of Variance (ANOVA) was used to compare changes in outcome measures over time.

Bootstrapping procedures (based on 5,000 samples) were used to estimate confidence intervals for indirect effects through the proposed mediators. This method was chosen as it makes no assumptions about the distribution of indirect effects and can be applied to small samples with a degree of confidence (Preacher \& Hayes, 2004). Significant mediator (indirect) effects are found when confidence intervals exclude zero. Variables were 
standardised before analysis to enable comparison of effect sizes. Moderator effects were examined via hierarchical regression incorporating interaction terms and simple slopes analyses (Aiken \& West, 1991). Moderator variables were centred before analysis to reduce essential multi-collinearity.

\section{Results}

\section{Intervention participation}

Eighty-five people were invited to take part in the intervention; 62 agreed and were randomised to either the Brief Intervention or the Control group; four ( 2 in each arm) did not use the MEMS pill bottle and were excluded from the analysis (Supplemental Figure S1). There were ceiling effects for the MARS scores with 59\% of participants taking part in the intervention having the maximum possible sub-optimal adherence score (i.e. MARS=24) - all reporting that they only 'rarely' forgot their medication. There were no differences between those in the Intervention versus the Control group on any of the socio-demographic variables or pre-treatment measures, suggesting the randomisation procedure was effective.

\section{Primary outcome measure - MEMS electronic pill bottle}

Results on the main outcome measure are fully reported in the simple effects article (O'Carroll et al., 2013). In brief, the Intervention group had higher overall adherence than the Control group on all MEMS measures (percentage of total doses taken: Intervention group mean 99\% versus Control 94\%, 95\% CI for difference $(-1.6,9.0)$; percentage of days correct dose taken: Intervention group mean 99\% versus Control 93\%, 95\% C.I.(-1.8, 9.4)), but this was only significant for percentage of doses taken on schedule (Intervention group mean 97\% versus Control mean $87 \%, 95 \%$ CI for difference $(0.2,16.2), p=.048)$. There were no time or treatment group by time interaction effects in any of the MEMS analyses.

\section{MARS Scores}

Figure 1 shows the changes in scores in self-reported adherence (MARS) from pre- 
intervention to follow-up. There were significant time (mean increase $0.62,95 \%$ C.I. (0.3, $0.9), p<.001$ ) and interaction effects (mean difference for Intervention minus Control 0.61, $95 \%$ C.I. for difference $(0.1,1.2), p=.027)$ for total MARS scores, with overall higher adherence at follow-up, but a significantly greater improvement in the Intervention group ${ }^{1}$.

Both non-intentional (i.e. MARS item 1) and intentional (i.e. MARS items 2-5) adherence also showed significant increases over time (non-intentional mean increase pre-treatment to follow-up $0.26,95 \%$ C.I. $(0.1,0.4), p<.001$; intentional mean increase $0.39,95 \%$ C.I. (0.1, $0.4), p<.001)$, and greater, though not significant, increases in the Intervention versus the Control group (non-intentional mean difference (Intervention minus Control) 0.15, 95\% C.I. $(-0.1,0.4), p=.138$; intentional mean difference $0.27,95 \%$ C.I. $(-0.2,0.7), p=.202)$.

\section{Beliefs about Medication and Illness}

Full details of between group differences on the BMQ, BIPQ and perceived benefit of medication are reported in the simple effects article (O'Carroll et al., 2013). In brief, BMQ necessity minus concerns significantly increased from pre-treatment to follow-up and BMQ concerns significantly decreased in both groups (see Supplemental Table S1). There was also

\footnotetext{
${ }^{1}$ Given the skewed nature of the MARS scores, non-parametric between group tests on
} MARS total scores were also conducted at pre-treatment (Mann-Whitney $U=478.5, p=$ $.975)$ and follow-up $(U=286.0, p=.020)$, as well as repeated measures on the MARS total scores from pre-treatment to follow-up for the Intervention group (Wilcoxon=153.0, $p<$ .001 ) and the Control group (Wilcoxon $=63.5, p=.185$ ) separately. These non-parametric tests support the findings of the repeated measures analysis above, with significantly higher self-reported MARS adherence in the Intervention arm at follow-up compared to the Control group. 
an interaction effect with BMQ concerns having a significantly greater decrease by follow-up in the Intervention versus the Control group (mean difference $1.3,95 \%$ CI $(0.1-2.5), p=$ .047). There were no significant effects of changes in beliefs about illness (BIPQ) or perceived benefit of medication as a result of the intervention.

\section{Predicting adherence: Regression analyses}

In order to examine whether changes in illness and medication beliefs as a result of the intervention were predictors of adherence, hierarchical regression analysis of changes in medication and illness beliefs from pre- to post-treatment on percentage of doses taken on schedule measured over the 3-month follow-up period was conducted (Table 1). The final model is significant $(F(11,44)=3.1, p=.004)$ and explains $44 \%$ of the variance in percentage doses taken on schedule (adjusted $\left.\mathrm{R}^{2}=30 \%\right)$, with older age $(\beta=.444, p=.002)$, smaller reductions $^{2}$ in BMQ concerns $(\beta=.335, p=.022)$, higher scores on the MMSE $(\beta=$ $.302, p=.036)$ and being in the Intervention treatment group $(\beta=-.293, p=.033)$ being significant predictors of higher adherence. Pooled results following multiple imputation showed similar effects, with pooled significant effects for age $(p=.006)$, MMSE $(p=.034)$ and change in BMQ concerns $(p=.025)$, although treatment group was no longer significant $(p=.108)$; amount of variance explained was $35-39 \%$ (adjusted $\left.\mathrm{R}^{2}=19-25 \%\right)$.

\footnotetext{
${ }^{2}$ This seems contrary to expectations, where greater (rather than smaller) reductions in concerns would be expected to relate to higher adherence. We propose that, as the Research Fellow was tasked with providing accurate, factual answers to patients' concerns, participating in the intervention may have heightened thinking about any negative effects of a patient's medication, resulting in a short-term increase in BMQ concerns for some participants. However, BMQ necessity minus concerns increased from pre- to post-treatment in the intervention, but not the control, group; this suggests that any increases in BMQ concerns in this group may have been outweighed by increases in BMQ necessity, which may have led to higher adherence at follow-up.
} 


\section{Mediators of treatment group effect}

The next step was to investigate whether the improved adherence in the Intervention group was mediated by changes in either beliefs and/or forgetting. A pre-requisite for a mediator variable is that all variables should have significant first-order correlations. The only variable meeting criteria for a mediator variable was change in BMQ concerns from post-treatment to follow-up, although change in forgetting (i.e. MARS item 1) from pre-treatment to follow-up was near to meeting criteria. Table 2 shows the indirect effects on percentage of doses taken on schedule and the regression coefficients for the $a, b, c$ and c' paths are shown in Figure 2.

The model was significant $(F(3,51)=5.1, p=.004$ and explained $23 \%$ of the variance in percentage of doses taken on schedule $\left(\mathrm{R}^{2}=.230\right.$; adjusted $\left.\mathrm{R}^{2}=.184\right)$. The results of the bootstrap analyses indicate that there was an overall significant mediation effect of both variables together $(95 \%$ C.I. $(-0.383,-0.031))$, as well as separate significant mediation effects of reduction in BMQ concerns from post-treatment to follow-up (95\% C.I. (-0.218, $0.007)$ ) and reductions in forgetting from pre-treatment to follow-up (95\% C.I. (-0.340, 0.002)). The effect of treatment group on percentage of doses taken on schedule controlling for reduction in both BMQ concerns and MARS forgetting was not significant (95\% C.I. ($.37, .14) ; p=.381)$, indicating that a moderate part of the effect of treatment group on percentage of doses taken on schedule could be explained by the indirect effect of these two variables. The contrast term for changes in BMQ concerns versus changes in forgetting indicates there was no difference in the magnitude of the effect of these two variables $(95 \%$ C.I. $(-0.115,0.249))$, meaning it is not possible to speculate as to which component of the intervention was more effective in increasing adherence. There were no other mediator effects of changes in illness or medication beliefs from pre- to post-treatment or from pretreatment to follow-up on any measures of adherence. 


\section{Moderators of treatment group effect}

At the beginning of session 1 the Research Fellow asked participants from both groups if they already had an established routine for medication taking, and recorded the number of Implementation Intentions cues (i.e. activity, time or place) spontaneously mentioned when describing this routine. Only one patient (2\%) reported having no routine, $12(21 \%)$ mentioned one Implementation Intentions cue (8 time and 4 activity), 42 (72\%) mentioned two cues and $3(5 \%)$ mentioned all three. The number of Implementation Intentions cues mentioned at first interview was significantly positively correlated with all MEMS adherence measures at follow-up (e.g., percentage of doses taken on schedule $(\mathrm{r}=.44, p=.001))$. A hierarchical liner regression analysis including treatment group at Step 1, number of pretreatment Implementation Intentions cues at Step 2, and the interaction of treatment group and number of Implementation Intentions cues at Step 3 was significant $(F(3,5)=7.1, p$ $<.001$ ) and explained $28.4 \%$ of the variance (adjusted $\mathrm{R}^{2}=24.4 \%$ ) in percentage of doses taken on schedule, with both number of Implementation Intentions cues $(\beta=-.37, p=.003)$ and the interaction $(\beta=.27, p=.025)$ being significant (but not treatment group; $\beta=.15, p=$ .213). This indicates that the number of initial Implementation Intentions cues reported by participants moderated the effect of treatment group on follow-up adherence. Simple slopes (Aiken \& West, 1991) were computed for the effect of treatment group on adherence at two levels of initial Implementation Intentions cues: low i.e. $<=1$ and high i.e. $>=2$ (Figure 3). Figure 3 shows that for low levels of initial Implementation Intentions cues, forming Implementation Intentions plans (i.e. being in the Intervention group) resulted in significantly higher adherence at follow-up compared to the Control group $(\beta=-1.2, p=.016)$, whereas there was no effect of treatment group for those with high initial levels of Implementation Intentions cues $(\beta=-0.2, p=.435)$. Cohen's $\mathrm{f}^{2}$ was used to estimate effect size: this estimates the ratio of systematic variance accounted for by the moderator relative to unexplained 
variance in the criterion (Aiken $\&$ West, 1991). For this analysis, $\mathrm{f}^{2}=.10$ indicating a small to medium effect of moderation (as defined by Cohen (1988): i.e. .02 is a small effect, and .15 is a medium effect).

\section{Discussion}

This pilot intervention is the first to show that adopting a simple If-then plan, coupled with eliciting and modifying patients' medication concerns, can significantly improve adherence to medication in stroke survivors. In contrast to some other studies with stroke survivors (e.g. Bushnell et al., 2010), overall adherence appeared to be relatively high. Reasons for this may include the fact this was an older, higher socio-economic sample (both of which are associated with higher adherence (O'Carroll et al., 2011)) or that many patients already had effective medication-taking routines (as evidenced by the high number of Implementation Intentions cues already used), which may be linked to the fact that many were on established multiple medications for chronic conditions. However, it is equally plausible that the high adherence represents a strong Hawthorne effect attributable to the use of the MEMS pill bottles over a three-month period. It seems likely that the use of MEMS pill bottles led to a significant increase in adherence in both treatment and control groups.

Despite the high adherence overall, the brief intervention improved the regularity of pilltaking by $10 \%$ over and above any non-specific effects of MEMS pill bottles and additional therapeutic contact. Although the corresponding 5\% increase in overall pills taken was not significant, instructions for taking common anti-hypertensives often advise taking medication at the same time each day, precisely to reduce forgetting (e.g. Lisinopril: 'Try to take your dose at the same time each day to avoid missing any doses'). Many of the patients in this study were also taking common cholesterol-lowering medications, where a delay in taking a dose should lead to a missed dose as indicated by the instructions (e.g. Simvastatin: 'If you miss a dose, just carry on with the next one as normal. Do not take an extra one to make up'). 
Therefore regularity of pill-taking may be just as important as numbers of doses taken in ensuring good clinical outcomes. Is a $10 \%$ increase in adherence clinically significant? Bailey, Wan, Tang, Ghani, and Cushman (2010) reported that increasing adherence to bloodpressure medication by just one tablet per week could reduce the hazard of stroke by $8-9 \%$ and of death by $7 \%$.

Overall, there was greater improvement in beliefs about medication (i.e. reduction in concerns) in the Intervention group, and evidence for a mediation effect of reduction in concerns about medication (from post-treatment to follow-up) and forgetting medication (from pre-treatment to follow-up) on objectively-measured adherence. Thus the dual goals of increasing adherence by improvements in both non-intentional non-adherence, through the process of reducing forgetting, and intentional adherence, via the process of reducing concerns about medication, was partly achieved. This intervention was tailored to each participant, and it has been suggested that simply tailoring to current behaviour and personal characteristics may have considerable effects on behaviour change (Noar, Benac, \& Harris, 2007). Participants in the present study were not given on feedback on their initial or subsequent BMQ scores; rather these were used to guide the interviewer's questions to help elicit specific concerns which could then be personally addressed. Participants frequently commented that it was beneficial to be able to get answers to their personal queries, in preference to the usual mass-produced media (such as Patient Information Leaflets). This, coupled with the changes in BMQ concern scores from pre-treatment to follow-up, suggests that changes in beliefs did contribute to increased adherence.

Consistent with previous research in stroke survivors (O'Carroll et al., 2011), illness perceptions were not related to adherence in the present study. The majority of this sample reported that they had no on-going symptoms from their stroke and thus viewed their illness as time-limited and/or 'over', and therefore as having no great impact on their lives or 
medication-taking. It is also possible that there was a bias in those taking part in the intervention towards having fewer lasting symptoms as a result of their stroke. However, it was not possible to explore this further from the current data.

Previous research aimed at increasing medication adherence has often had limited success (Haynes, Ackloo, Sahota, McDonald, \& Yao, 2008); the present study adds to current evidence by demonstrating that a brief intervention using a combined approach to simultaneously address both reflective and automatic aspects of behaviour can increase medication adherence in a population of elderly stroke survivors. These findings support temporal self-regulation theory in that implementation intentions formation may be viewed as a self-regulatory tool, which can help individuals with cognitive impairment attain their goals via developing automatic processes, whilst the aim of addressing any dysfunctional beliefs was to alter patients' views of the cost-benefit equation in favour of taking their medication on a regular basis.

Many of the patients had been on blood pressure medicine and/or other long-term medication before their stroke or TIA, and many had already established regular medicationtaking routines. The number of Implementation Intentions cues spontaneously mentioned at the first interview was strongly related to MEMS adherence at follow-up, and also moderated the effect of treatment group, such that the effect of the intervention was mainly apparent in patients with a poorly-defined initial routine (i.e. had weak positive habits). This could be because they benefited from help in setting up a routine, or because those with a wellestablished routine may have been less amenable to change it, even when it was not always working successfully. Lally and Gardner (2011) reasoned that deliberate intentions to perform a behaviour have a reduced influence when habits are already strongly established. The current findings lend further support to temporal self-regulation theory, where past behaviour is viewed as an important determinant of future behaviour. They also indicate that 
Implementation Intentions planning is likely to be most effective at increasing adherence in patients who are new to medication-taking, or have failed to establish a regular routine by their own devices, suggesting that early intervention may be key.

\section{Strengths and Limitations}

A critical evaluation of adherence interventions (Horne, Weinman, Barber, Elliot, \& Morgan, 2006) identified six consistent weaknesses in the field: 1) narrow focus for intervention, in particular a failure to consider both intentional and non-intentional non-adherence, 2) "one size fits all" approach i.e. not patient-centred, 3) failure to specify the content of the intervention, 4) "black box" evaluation, 5) lack of theoretical framework and 6) little or no process evaluation. Our study has addressed all of these issues.

The main limitation of this pilot study was that the Research Fellow was not blinded to treatment arm of the patients, as she both delivered the intervention and carried out the analysis. However, this should not have had any major effect on the current findings as the primary outcome measure was objectively-recorded electronic MEMS readings, and the remaining outcome measures were posted out to and completed by participants in advance of the Research Fellow visits. Baseline data was not recorded for the MEMS; however there were no difference in pre-treatment self-reported adherence between groups, and selfreported adherence also showed greater increases in the Intervention versus the Control group, suggesting the differences found in MEMS readings would not have been present at pre-treatment. Nonetheless, it would have been preferable to have a run-in period with the MEMS pill bottle to establish baseline adherence on the objective measure; however, this was not feasible within the scope of this pilot study.

The high levels of adherence observed in both groups in the current study, plus the fact that those reporting they already used two or more Implementation Intentions cues had higher adherence at follow-up in both treatment arms, suggests that the intervention may have had 
greater impact had the intervention been targeted at participants with lower initial levels of adherence. Additional limitations include the small sample size and the fact that there was only one item measuring non-intentional non-adherence. Although this pilot was conducted in stroke survivors, it is plausible that it may be generalizable to other patients on preventive medication for chronic conditions.

\section{Conclusions}

To our knowledge, no other published study has used an intervention aimed at increasing adherence in a group of stroke survivors via helping them devise medication-taking routines in conjunction with aiming to elicit and address any mistaken medication and/or illness beliefs. These findings demonstrate that a simple, individually tailored, theory-based intervention resulted in a significant increase in objectively measured adherence over a 3month period. In addition, it would appear that the combined approach of addressing both automatic and reflective behaviour was instrumental in the outcome, as the treatment effect was mediated via a reduction in both forgetting and concerns about medications, and moderated by the degree of pre-existing medication-taking routines.

\section{Conflicts of Interest.}

There are no conflicts of interest.

\section{Acknowledgements.}

This project was funded by a grant from the Scottish Government, Department of Health, Chief Scientist Office, reference number $\mathrm{CZH} / 4 / 569$. We would like to thank the doctors and nurses at the Western General Hospital stroke clinic and ward for their help in recruitment, and the participants for giving up their time to take part. 


\section{References}

Aiken, L. S., \& West, R. R. (1991). Multiple regression: testing and interpreting interactions. Newbury Park, CA: Sage.

Alhalaqia, F., Nawafleh, A. H., Clark, A., \& Gray, R. (2012). Adherence therapy for medication non-compliant patients with hypertension: a randomised controlled trial. Journal of Human Hypertension, 26, 117-126.

Anthony, H., Valinsky, L., Inbar, Z., Gabriel, C., \& Varda, S. (2012). Perceptions of hypertension treatment among patients with and without diabetes. BMC Family Practice, $13,24$.

Bailey, J. E., Wan, J. Y., Tang, J., Ghani, M. A., \& Cushman, W. C. (2010). Antihypertensive medication adherence, ambulatory visits, and risk of stroke and death. Journal of General Intern Medicine, 25, 495-503.

Broadbent, E., Petrie, K. J., \& Weinman, J. (2004). The Brief Illness Perception Questionnaire (BIPQ): Validity and Reliability. International Journal of Behavioral Medicine, 11, 278.

Brown, I., Sheeran, P., \& Reuber, M. (2009). Randomized Controlled Trial of an Implementation Intention Intervention to Enhance Adherence with Antiepileptic Drug Treatment. Epilepsy \& Behaviour, 16, 634-639.

Bushnell, C. D., Zimmer, L. O., Pan, W., Olson, D. M., Zhao, X., Meteleva, T., .. Peterson, E. D. (2010). Persistence with stroke prevention medications 3 months after hospitalization. Archives of Neurology, 67(12), 1456-63.

Chambers, J. A., O’Carroll, R. E., Hamilton, B., Whittaker, J., Johnston, M., Sudlow, C., \& Dennis, M. (2011). British Journal of Health Psychology, 16, 592-609.

Clifford, S., Barber, N., \& Horne, R. (2008). Understanding different beliefs held by adherers, unintentional nonadherers, and intentional non-adherers; Application of the Necessity-Concerns Framework. Journal of Psychosomatic Research, 64, 41-46.

Cohen, J. (1988). Statistical power analysis for the behavioral sciences (2nd ed.). Hillsdale, NJ: Erlbaum.

Folstein, M. F., Folstein, S. E., \& McHugh, P. R. (1975). "Mini-mental state". A practical method for grading the cognitive state of patients for the clinician. Journal of Psychiatric Research, 12, 189-198.

Gollwitzer, P. M. (1999). Implementation intentions: Strong effects of simple plans. American Psychologist, 54, 493-503. 
Hall, P. A., \& Fong, G. T. (2007). Temporal self-regulation theory: A model for individual health behavior. Health Psychology Review, 1, 6-52.

Hayes, T. L., Larimer, N., Adami, A., \& Kaye, J. A. (2009). Medication adherence in healthy elders: small cognitive changes make a big difference. Journal of Aging and Health, 21(4), 567-580.

Haynes, R. B., Ackloo, E., Sahota, N., McDonald, H. P., \& Yao, X. (2008). Interventions for enhancing medication adherence. Cochrane Database of Systematic Reviews, Issue 2. Art. No.: CD000011. doi: 10.1002/14651858.CD000011.pub3.

Horne, R. (2004). Measuring adherence: the case for self-report. International Journal of Behavioral Medicine, 11, 75.

Horne, R., \& Weinman, J. (1999). Patients' beliefs about prescribed medicines and their role in adherence to treatment in chronic physical illness. Journal of Psychosomatic Research, $47,555-567$.

Horne, R., Weinman, J., Barber, N., Elliot, R. A., \& Morgan, M. (2006). Concordance, Adherence and Compliance in Medication Taking: a conceptual map and research priorities. London: Centre for NHS Service Delivery and Organisation NCSSDO.

Horne, R., Weinman, J., \& Hankins, M. (1999). The Beliefs about Medicines Questionnaire: The development and evaluation of a new method for assessing the cognitive representation of medication. Psychology \& Health, 14, 1-24.

Lally, P., \& Gardner, B. (2011). Promoting habit formation. Health Psychology Review, 7, Supplement 1, S137-S158.

Liu, L. L., \& Park, D. C. (2004). Aging and medical adherence: The use of automatic processes to achieve effortful things. Psychology and Aging, 19, 318-325.

Meyer, D., Leventhal, H., \& Gutmann, M. (1985). Common-sense models of illness: the example of hypertension. Health Psychology, 4, 115-135.

Michie, S., Richardson, M., Johnston, M., Abraham, C., Francis, J., Hardeman, W., . . . Wood, C. E. (2013). The Behavior Change Technique Taxonomy (v1) of 93 Hierarchically Clustered Techniques: Building an International Consensus for the Reporting of Behavior Change Interventions. Annals of Behavioral Medicine, 46, 81-95.

Moher, D., Hopewell, S., Schulz, K. F., Montori, V., Gøtzsche, P. C., Devereaux, P. J., . . . Altman, D. G. (2010). CONSORT 2010 Explanation and Elaboration: Updated guidelines for reporting parallel group randomised trials. British Medical Journal, 340, c869. doi:10.1136/bmj.c869 
Noar, S. M., Benac, C. N., \& Harris, M. S. (2007). Does tailoring matter? Meta-analytic review of tailored print health behavior change interventions. Psychological Bulletin, 133(4), 673-693.

O’Carroll, R.E., Chambers, J.A., Dennis, M., Sudlow, C., Johnston, M. (2013). Improving adherence to medication in stroke survivors: a pilot randomised controlled trial. Annals of Behavioral Medicine, 46, 358-368.

O'Carroll, R. E., Dennis, M., Johnston, M., \& Sudlow, C. (2010). Improving Adherence to Medication in Stroke Survivors (IAMSS): a randomised controlled trial: study protocol. BMC Neurology, 10, 15.

O'Carroll, R. E., Whittaker, J., Hamilton, B., Johnston, M., Sudlow, C., \& Dennis, M. (2011). Predictors of adherence to secondary prevention medication in stroke patients. Annals of Behavioral Medicine, 41, 383-90.

Petrie, K. J., Cameron, L. D., Ellis, C. J., Buick, D., \& Weinman, J. (2002). Changing illness perceptions after myocardial infarction: an early intervention randomized controlled trial. Psychosomatic Medicine, 64, 580-586.

Pound, P., Britten, N., Morgan, M., Yardley, L., Pope, C., Daker-White, G., \& Campbell, R. (2005). Resisting medicines: a synthesis of qualitative studies of medicine taking. Social Science \& Medicine, 61, 133-155.

Preacher, K. J., \& Hayes, A. F. (2004). SPSS and SAS procedures for estimating indirect effects in simple mediation models. Behavior Research Methods Instruments \& Computers, 36, 717-731.

Roger, V. L., Go, A. S., Lloyd-Jones, D. M., Benjamin, E. J., Berry, J. D., Borden, W. B., . . . Turner, M. B. (2012). Heart Disease and Stroke Statistics - 2012 update. A report from the American heart Association. Circulation, 125: e2-e220.

Rubin, D. B. (1987). Multiple imputation for non-response in surveys. New York: John Wiley.

Sniehotta, F. F., Scholz, U., \& Schwarzer, R. (2006). Action plans and coping plans for physical exercise: A longitudinal intervention study in cardiac rehabilitation. British Journal of Health Psychology, 11, 23-37.

Strack, F., \& Deutsch, R. (2004). Reflective and impulsive determinants of behaviour. Personality and Social Psychology Review, 8, 220-247.

Sudlow, C. (2008). Preventing further vascular events after a stroke or transient ischaemic attack: an update on medical management. Practical Neurology, 8, 141-157. 
Trewby, P. N., Reddy, A. V., Trewby, C. S., Ashton, V. J., Brennan, G., \& Inglis, J. (2002). Are preventive drugs preventive enough? A study of patients' expectation of benefit from preventive drugs. Clinical Medicine, 2, 527-533.

Warlow, C., Sandercock, P., Hankey, G., van Gijn, J., Dennis, M., Bamford, J., . . Rothwell, P. (2008). Stroke: Practical Management, 3rd ed. Oxford: Blackwell.

Webb, T. L., Sheeran, P., \& Luszczynska, A. (2009). Planning to break unwanted habits: Habit strength moderates implementation intention effects on behaviour change. British Journal of Social Psychology, 48, 507-23.

WHO. (2003). Adherence to long term therapies: evidence for action. Geneva: World Health Organisation. 
Table 1. Multiple hierarchical linear regression predicting percentage of doses taken on schedule (full data: $\boldsymbol{n}=57$ )

\begin{tabular}{|c|c|c|c|c|c|c|c|c|c|}
\hline & & $\begin{array}{c}\beta \\
\text { Step } 1\end{array}$ & $\begin{array}{c}\beta \\
\text { Step } 2\end{array}$ & $\begin{array}{c}\beta \\
\text { Step } 3\end{array}$ & $\begin{array}{c}\beta \\
\text { Step } 4\end{array}$ & $\begin{array}{c}\beta \\
\text { Step } 5\end{array}$ & $\begin{array}{c}\text { Change } \\
R^{2} \text { for } \\
\text { step }\end{array}$ & Total $R^{2}$ & $\begin{array}{c}\text { Adjusted } \\
R^{2}\end{array}$ \\
\hline \multirow[t]{4}{*}{1} & Demographics & & & & & & $.188 * *$ & $.188 * *$ & .151 \\
\hline & Age & $.310 *$ & $.402 * *$ & $.396^{*}$ & $.381 * *$ & $.444 * *$ & & & \\
\hline & Gender & -.115 & -.152 & -.155 & -.277 & -.236 & & & \\
\hline & SIMD & -.236 & -.188 & -.184 & -.256 & -.196 & & & \\
\hline \multirow[t]{3}{*}{2} & Stroke severity & & & & & & .051 & $.239 *$ & .163 \\
\hline & MMSE Total Score & & .211 & 205 & $.348^{*}$ & $.302 *$ & & & \\
\hline & Severity & & .113 & .107 & .105 & .071 & & & \\
\hline \multirow[t]{3}{*}{3} & Illness perceptions & & & & & & .002 & $.240 *$ & .130 \\
\hline & BIPQ Treatment Control pre-post & & & .027 & .083 & .007 & & & \\
\hline & BIPQ Timeline pre-post & & & .041 & -.009 & -.080 & & & \\
\hline \multirow[t]{4}{*}{4} & Medication beliefs & & & & & & $.135 *$ & $.375^{*}$ & .236 \\
\hline & BMQ Specific Necessity pre-post & & & & .181 & .105 & & & \\
\hline & BMQ Specific Concerns pre-post & & & & $.386^{*}$ & $.335^{*}$ & & & \\
\hline & Perception of Medication Benefits & & & & .080 & .146 & & & \\
\hline \multirow[t]{2}{*}{5} & Treatment Group & & & & & & $.062 *$ & .437 & .297 \\
\hline & Treatment group & & & & & $-.293 *$ & & & \\
\hline
\end{tabular}

SIMD: Scottish Index of Multiple Deprivation; MMSE: Mini Mental State Exam; BIPQ: Brief Illness Perception Questionnaire;

BMQ: Beliefs about Medication Questionnaire. ${ }^{*} p<.05$. $* * p<.01$. 
Table 2. Mediation of the effect of treatment on percentage of doses taken on schedule through reduction in forgetting and BMQ concerns from post-treatment to follow-up

\begin{tabular}{|l|c|c|c|c|c|}
\hline & Point estimate & Product of coefficients & \multicolumn{2}{|c|}{ Bca 95\% C.I. } \\
\hline & & $\mathrm{SE}$ & $\mathrm{Z}$ & Lower & Upper \\
\hline BMQ concerns from post-treatment to follow-up & -.067 & .048 & -1.43 & -.218 & -.007 \\
\hline Forgetting (MARS item 1) & -.082 & .070 & -1.17 & -.340 & -.002 \\
\hline Total & -.151 & .085 & -1.77 & -.383 & -.031 \\
\hline Contrast (BMQ versus forgetting) & .013 & .085 & 0.16 & -.115 & .249 \\
\hline
\end{tabular}

$\mathrm{BMQ}=$ Beliefs about Medication Questionnaire; $\mathrm{Bca}=$ bias corrected and accelerated bootstrapping confidence intervals; intervals are significant if they do not contain zero (Preacher \& Hayes, 2004). 


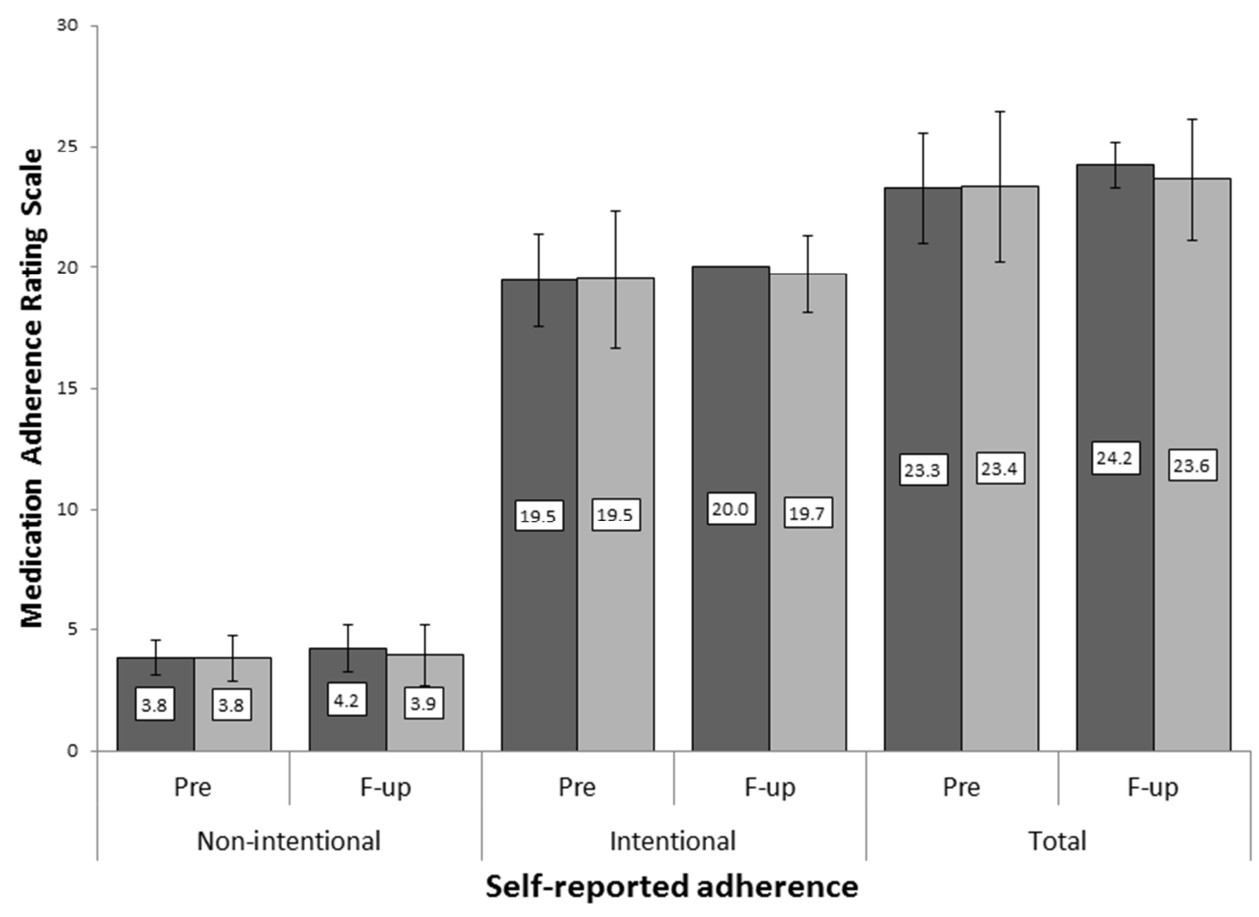

Note: Error bar for intentional adherence at follow-up for the Intervention group $=20$ (all scores=20)

Figure 1. Total scores on the Medication Adherence Report Scale (MARS) from pre-treatment to 3-month follow-up (Error bars represent 95\% C.I. for mean) 


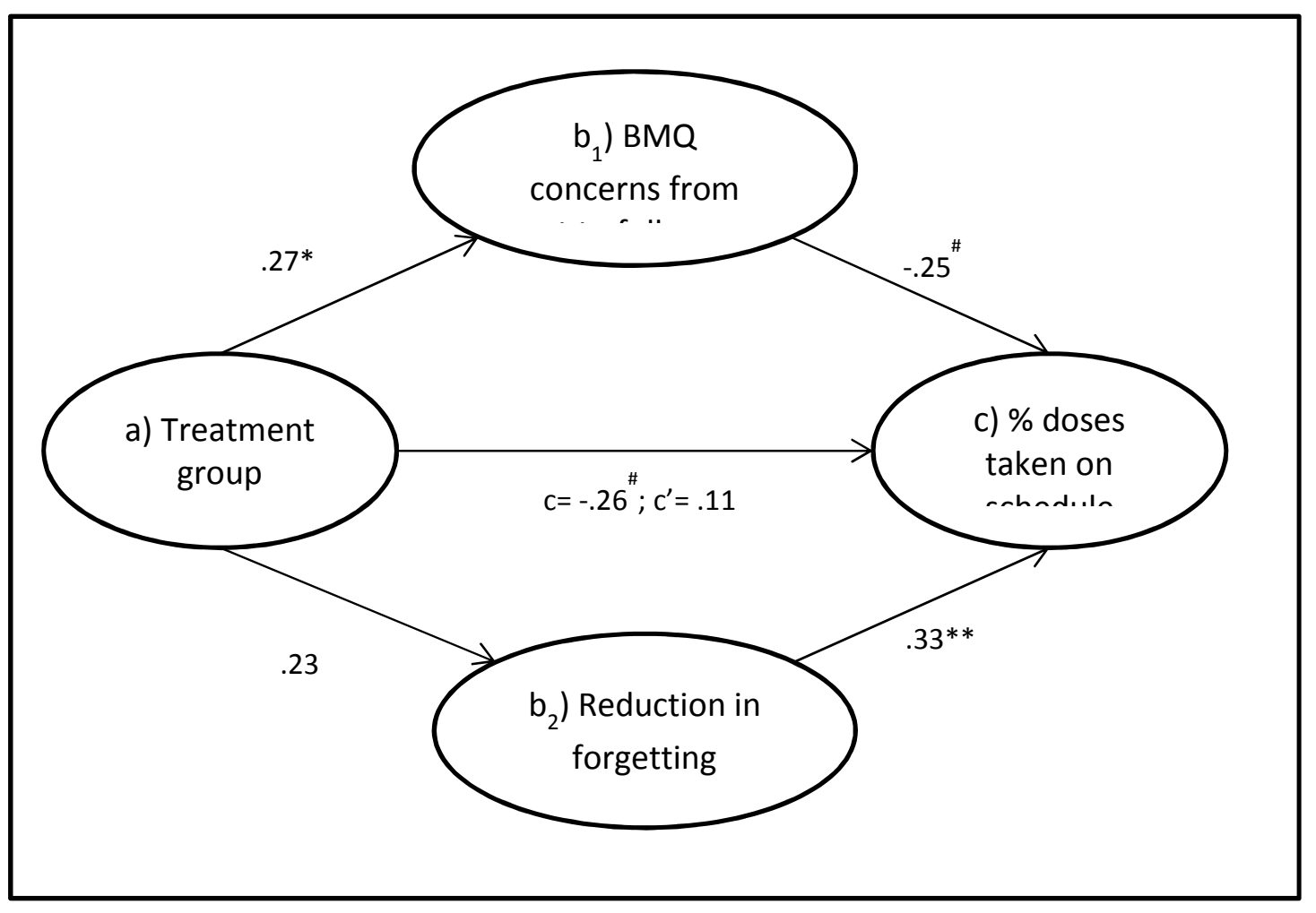

\# $<.06, * p<.05, * * p<.01 ; B M Q=$ Beliefs about Medication Questionnaire

Figure 2. Effects of treatment group on percentage of doses taken on schedule by changes in medication beliefs and forgetting 


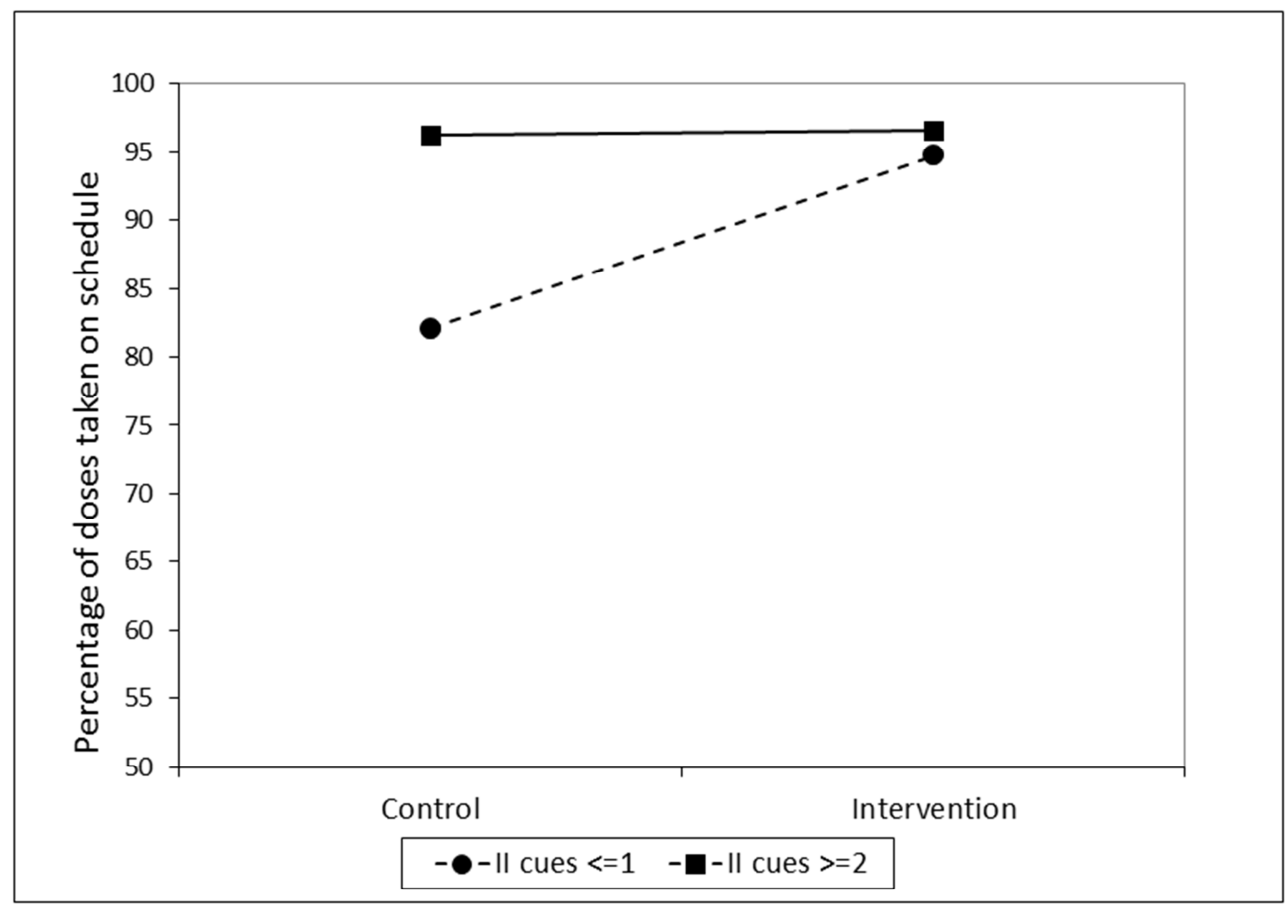

Figure 3. Simple slopes analysis for the moderator effect of number of initial Implementation Intentions (II) cues on the relationship between treatment group and percentage of doses taken on schedule 


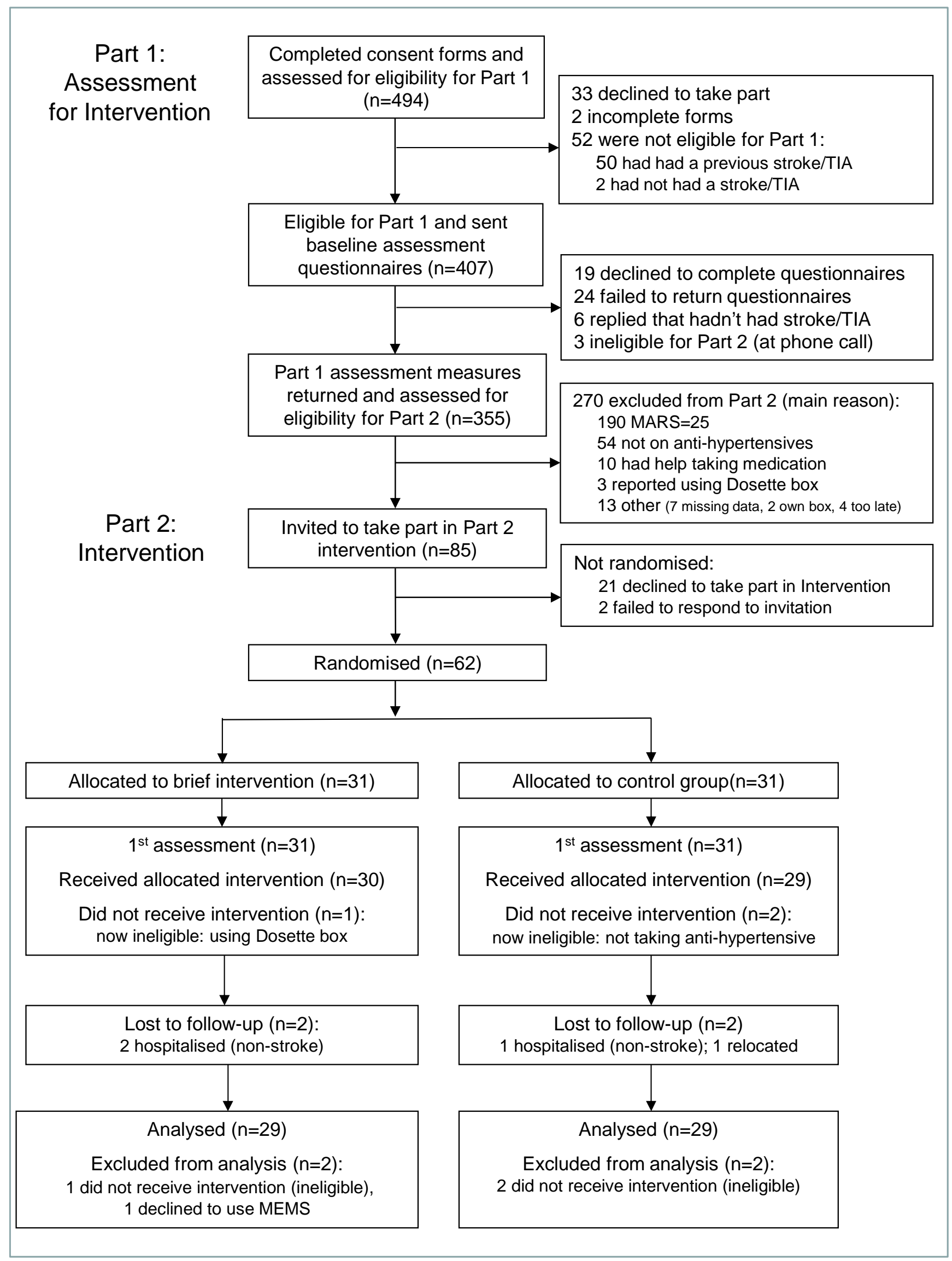

\section{Supplemental Figure S1. CONSORT diagram}


Supplemental Table S1. Mean scores (s.d.) and results of ANOVAs for Beliefs about Medication by time and treatment group (n=58)

\begin{tabular}{|c|c|c|c|c|c|c|c|}
\hline & & & & & Group $x$ time & Group & Time \\
\hline & & Pre & Post & Follow-up & Pooled $F(2, x)$ & Pooled $F(1, x)$ & Pooled $F(1, x)$ \\
\hline \multirow[t]{2}{*}{ BMQ Specific Necessity } & Intervention & $17.4(3.0)$ & $18.1(2.6)$ & $18.5(2.5)$ & \multirow{2}{*}{$0.8, p=.460$} & \multirow{2}{*}{$0.1, p=.743$} & \multirow{2}{*}{ 2.7, $p=.066$} \\
\hline & Control & $18.2(3.0)$ & $17.9(3.0)$ & $18.5(2.7)$ & & & \\
\hline \multirow[t]{2}{*}{ BMQ Specific Concerns } & Intervention & $13.2(3.2)$ & $11.9(3.7)$ & $11.0(2.5)$ & \multirow{2}{*}{$3.1, p=.047$} & \multirow{2}{*}{$.01, p=.924$} & \multirow{2}{*}{$9.2, p<.001$} \\
\hline & Control & $12.9(3.4)$ & $11.7(3.7)$ & $11.9(3.0)$ & & & \\
\hline \multirow[t]{2}{*}{ BMQ Necessity minus Concerns } & Intervention & $4.2(4.5)$ & $6.1(4.9)$ & $7.4(3.4)$ & \multirow{2}{*}{$2.5, p=.081$} & \multirow{2}{*}{$0.02, p=.879$} & \multirow{2}{*}{$10.8, p<.001$} \\
\hline & Control & $5.3(4.6)$ & $6.2(4.9)$ & $6.6(4.3)$ & & & \\
\hline
\end{tabular}

Note: df for F (i.e. $\mathrm{x}$ ) are adjusted by inter-imputation variance to give pooled p-values and so vary by each statistic;

BMQ: Beliefs about Medication Questionnaire 